\title{
Crecimiento del pez Plagioscion squamosissimus (Perciformes: Sciaenidae) según la inferencia de modelos múltiples en la cuenca del Orinoco medio, Venezuela
}

Ángel González ${ }^{1} \&$ Arístide Márquez ${ }^{2 *}$

1. Instituto Limnológico, Universidad de Oriente, Núcleo de Bolívar, Venezuela; angelgonzalez78@hotmail.com

2. Instituto Oceanográfico de Venezuela, Oceanografía Química, Universidad de Oriente, Núcleo de Sucre, Avenida San Luis, Cerro Colorado, Cumaná, Estado Sucre, Venezuela; aristd@gmail.com

* Correspondencia

\author{
Recibido 09-IX-2019. Corregido 07-III-2020. Aceptado 05-VI-2020.
}

\begin{abstract}
Growth of the fish Plagioscion squamosissimus (Perciformes: Sciaenidae) according to multiple models inference in the middle Orinoco basin, Venezuela. Introduction: The prior use of von Bertalanffy's model has been generally used in the study of fish growth, without considering the existence of other growth models that can produce a better adjustment of the data used; considering the use of multiple growth models as a better alternative, to select the one that produces a better adjustment. Objective: Due to uncertainty of the results obtained in a previous work on the growth of Plagioscion squamosissimus (Heckel 1840 ) in the middle Orinoco region in Venezuela, applying a priori the model of von Bertalanffy, the same data were adjusted to other models of growth to select the best adjustment. Methods: The models evaluated were the U-von Bertalanffy, the U-Logistics and the U-Gompertz, derived from the global U-Richards model, then the best adjustment was selected using the Akaike Information Criteria $(A I C)$. Results: Contradicting the prior use of von Bertalanffy's model in the previous study, the model that actually produces a better adjustment of the data used was the U-Gompertz. Conclusions: It is necessary to evaluate the stock of $P$. squamosissimus in the middle Orinoco region using growth parameters estimated from an inference of multiple models, in order to validate the existing information of a moderately exploited resource.
\end{abstract}

Key words: river fish; Plagioscion squamosissimus; Middle Orinoco; population dynamics; growth; information theory.

González, A. \& Márquez, A. (2020). Crecimiento del pez Plagioscion squamosissimus (Perciformes: Sciaenidae) según la inferencia de modelos múltiples en la cuenca del Orinoco medio, Venezuela. Revista de Biología Tropical, 68(3), 892-897.

La curvinata de río Plagioscion squamosissimus es una de las especies de mayor desembarco en la región del Orinoco medio junto con el bagre rayado Pseudoplatystoma orinocoense ( $P$. fasciatum) y ocasionalmente el coporo Prochilodus mariae y el bagre dorado Brachyplatystoma rousseauxii (González, Mendoza, Arocha, \& Márquez, 2016). Esto ha ocasionado un incremento de la presión de pesca durante los últimos años, que aparentemente ha provocado una sobreexplotación del recurso. Una evaluación anterior del stock demostró un nivel de explotación moderado de P. squamosissimus en la región del Orinoco medio (González, Mendoza, Arocha, \& Márquez, 2005b). Sin embargo, esta afirmación ha generado cierta incertidumbre por haberse derivado a partir de parámetros de crecimiento 
provenientes de la selección a priori del modelo de von Bertalanffy (González, Mendoza, Arocha, \& Márquez, 2005a), al asumirlo como un modelo verdadero en el ajuste de los datos utilizados, lo cual es una suposición poco realista y no justificada desde el punto de vista matemático (Costa, Barthem, Albernaz, Bittencourt, \& Villacorta-Corrêa, 2013).

Contrario a la selección a priori del modelo de von Bertalanffy para el estudio del crecimiento de los peces, durante los últimos años se ha considerado como mejor alternativa la inferencia de modelos múltiples de crecimiento y el uso del AIC para seleccionar el de mejor ajuste (Katsanevakis \& Maravelias, 2008; Costa et al., 2013; Ansah \& Frimpong, 2015; Aragón-Noriega, Alcántara-Razo, ValenzuelaQuiñonez, \& Rodriguez-Quiroz, 2015). Un enfoque aplicado en el presente trabajo para validar los resultados obtenidos por González et al. (2005a) en cuanto al crecimiento de $P$. squamosissimus en el Orinoco medio.

En la inferencia de modelos múltiples se han usado generalmente las formas tradicionales de los modelos de von Bertalanffy, Logístico y Gompertz, entre otros, los cuales presentan limitaciones por interpretar de manera diferente los parámetros de crecimiento, y consecuentemente restricciones en la comparación de resultados. Una mejor opción ante el uso de estos modelos tradicionales es utilizar el modelo Unificado de la familia U-Richards, que permite la estimación de parámetros que pueden ser interpretados y comparados de manera coherente (Tjørve \& Tjørve, 2010; Tjørve \& Tjørve, 2017).

El modelo unificado de la familia U-Richards incluye los modelos U-von Bertalanffy, U-Logístico y U-Gompertz. Estos modelos fueron utilizados en el presente trabajo para el ajuste de los datos de longitud-edad usados por González et al. (2005a) en el estudio del crecimiento de $P$. squamosissimus en una laguna de inundación y en el canal principal del Orinoco medio (González et al., 2005a). Entre estos modelos se seleccionó el de mejor ajuste usando el AIC, y se verificó si realmente el modelo de von Bertalanffy usado por González et al. (2005a) es el que mejor se acomoda a los datos utilizados, como estos autores supusieron al usar a priori dicho modelo. De esta forma, se validaron igualmente los valores de los parámetros de crecimiento estimados por dicho modelo y su posterior uso en la evaluación de $P$. squamosissimus en el Orinoco medio, que dio como resultado la existencia de una población moderadamente explotada (González et al., 2005b).

\section{MATERIALES Y MÉTODOS}

Área de estudio: Los datos de longitudedad utilizados en este trabajo fueron extraídos de González et al (2005a), quienes estudiaron el crecimiento de $P$. squamosissimus en una laguna de inundación y en el canal principal de la parte media del río Orinoco; específicamente en la región de Caicara del Orinoco del estado Bolívar en Venezuela (7³9’00" N - 66010’00” W).

Metodología: Considerando que el crecimiento de $P$. squamosissimuses es igual en las lagunas de inundación y en el canal principal del Orinoco medio (González et al., 2005a), se consideró un solo conjunto de datos para la región. Se agruparon en dos subconjuntos que incluyeron 462 datos obtenidos por el procedimiento del retro-cálculo de la longitud de los peces a la formación de los anillos de crecimiento en los otolitos. Además de 903 datos obtenidos a partir de la distribución de frecuencia de longitudes de los peces, descompuestas en sus respectivas cohortes (edad) mediante el método de Battacharya.

Los dos subconjuntos de datos de longitud-edad, conformados por datos observados y retro-calculados, fueron ajustados a los modelos de crecimiento de U-von Bertalanffy, U-Logístico y U-Gompertz, incluidos en la familia U-Richards, y seleccionado el de mejor ajuste mediante el Criterio de la Información de Akaike (AIC).

El modelo global U-Richards estuvo representado por las siguientes ecuaciones 


$$
\begin{gathered}
L(t)=L_{\infty}(1+(d-1)) * \exp \left(\frac{-K\left(t-T_{i}\right)}{\frac{d}{d^{(1-d)}}}\right)^{\frac{1}{1-d}} \mathrm{y} \\
L_{(t)}=L_{\infty}\left(1+\left(\left(\frac{W_{o}}{L_{\infty}}\right)^{(1-d)}-1\right) * \exp \left(\frac{-K t}{\frac{d}{d^{1-d}}}\right)\right)^{\frac{1}{1-d}} ;
\end{gathered}
$$

a partir del cual se derivó el modelo U-von Bertalanffy dándole a $d$ un valor de $2 / 3(d=2 / 3)$ y el modelo U-Logístico otorgándole a $d$ un valor de dos $(d=2)$ (Tjørve \& Tjørve, 2010; Tjørve \& Tjørve, 2017).

El modelo U-Gompertz también se derivó del modelo global U-Richards pero $d$ se calculó como un límite, sin darle valor alguno, utilizando las ecuaciones

$$
\begin{gathered}
L_{(t)}=L_{\infty} * \exp \left(-\exp \left(-e * K\left(t-T_{i}\right)\right)\right) \\
\mathrm{y} \\
L_{(t)}=L_{\infty}\left(\frac{L_{\infty}}{W_{o}}\right)
\end{gathered}
$$

(Tjørve \& Tjørve, 2017).

En estas ecuaciones, $L_{(t)}=$ longitud teórica; $L_{\infty}=$ longitud asintótica; $t=$ edad; $K=$ tasa máxima de crecimiento relativo en la inflexión de la curva; $T_{i}=$ edad en la inflexión de la curva; $d=$ exponente o parte del mismo que controla el valor en la inflexión; $W_{o}=$ valor inicial de la curva $(t=0) ; e=$ número neperiano (González, Mendoza, Arocha, \& Márquez, 2019).

La clasificación y comparación de los modelos para seleccionar el de mejor ajuste se hizo utilizando el AIC según las ecuaciones

$$
\begin{gathered}
A I C_{c}=n * \log \left(\sigma^{2}\right)+2 k+\frac{2 k+(k+1)}{n-k-1} \\
\Delta A I C_{c}=A I C_{c}-A I C_{\min }
\end{gathered}
$$

donde $A I C_{c}$ fue el AIC de cada modelo,

$$
\sigma^{2}=\frac{R S S}{n},
$$

RSS la suma de cuadrado residual de cada modelo, $n$ el tamaño de la muestra, $k$ el número total de parámetros de regresión estimados en cada modelo (incluyendo a $\sigma^{2}$ ). $A I C_{\min }$ fue el $A I C_{c}$ más pequeño y $\triangle A I C_{c}$ el grado de separación de cada $A I C_{c}$ con relación al $A I C_{\text {min }}$.

El modelo más preciso en el ajuste de los datos fue aquel que presentó el valor mínimo del AIC $\left(A I C_{\min }\right)$, considerándose que mientras mayor sea la diferencia entre el AIC de cada modelo $\left(A I C_{c}\right)$ con relación al $A I C_{\text {min }}\left(\triangle A I C_{c}\right)$ menos probable es el modelo de producir un buen ajuste. En cuanto a esto se consideró que los modelos donde el $\triangle A I C_{c}$ es menor que dos $\left(\triangle A I C_{c}<2\right)$ producen un buen ajuste de los datos, cuando tiene valores entre cuatro y siete $\left(4 \leq \triangle A I C_{c} \leq 7\right)$ los ajusta medianamente, $\mathrm{y}$ cuando es mayor que diez $\left(\triangle A I C_{c}>10\right)$ esencialmente no producen un buen ajuste (Costa et al., 2013; Burnham \& Anderson, 2002; ArzolaSotelo, 2013).

Para certificar la selección del mejor modelo se utilizó la ponderación de Akaike (Wi), según la ecuación

$$
\frac{e^{\left(-\frac{1}{2} * \Delta i\right)}}{\sum e^{\left(-\frac{1}{2} * \Delta i\right)}}
$$

donde $\Delta i$ fue el $\Delta \mathrm{AIC}_{c}$ de cada modelo. Según el valor de $W i$ se consideró como el modelo de mejor ajuste aquel cuyo valor fue mayor que $0.9(W i>90 \%)$, mientras que cuando los valores de Wi fueron menor que 0.9 (Wi $<90$ $\%)$ se supuso que ningún modelo fue superior a otro; procediéndose en estos casos a determinar un modelo promedio, cuyos parámetros se estimaron por la ecuación $X_{\text {prom }}=\sum W i * X_{i}$, con un error estándar determinado por la ecuación E.S. $X_{\text {prom }}=\sum \mathrm{Wi}^{*}\left(\operatorname{Var} X_{i}+\left(X_{i}-X_{\text {prom }}\right)^{2}\right)^{1 / 2}$, donde E.S. $X_{\text {prom }}$ es el error estándar del parámetro $X$, y $\operatorname{Var} X_{i}$ la varianza del parámetro $X$ (González et al., 2019).

\section{RESULTADOS}

Los estimados de los parámetros de crecimiento $L_{\infty}, K, T_{i}$ y $W_{o}$, los estadísticos $R^{2}$ y $R S S$, y los resultados del AIC $\left(A I C_{c}, \Delta \mathrm{AIC}_{c} \mathrm{y}\right.$ $W_{\mathrm{i}}$ ) para los dos grupos de datos, se encuentran representados en el Cuadro 1; donde se observa que usando el coeficiente de determinación $\left(R^{2}\right)$ como indicativo de un buen ajuste de datos, como se hace frecuentemente, los tres modelos produjeron relativamente un buen ajuste. Sin embargo, según el valor mínimo del AIC 
$\left(A I C_{\text {minimo }}\right)$ para los dos grupos de datos, el modelo más preciso en el ajuste correspondió al de U-Gompertz (Cuadro 1) y por lo tanto fue realmente el modelo más preciso en el ajuste de los datos; obteniéndose un grado de separación ( $\triangle \mathrm{AICc}$ ) de los modelos U-von Bertalanffy y U-Logístico con relación al modelo más preciso (U-Gompertz) mayor que diez ( $\triangle \mathrm{AICc}>$ $10)$, que indicó un no buen ajuste de los datos por parte de ambos modelos. El mejor ajuste producido por el modelo U-Gompertz también fue corroborado por el valor de $W_{i}\left(W_{i}=1.0\right)$, según el cual dicho modelo produjo un 100 $\%$ de ajuste (Cuadro 1); siendo nulo el ajuste de los otros dos modelos $\left(W_{i}=0.0\right)$. En vista de la superioridad en cuanto al ajuste de los datos por parte del modelo U-Gompertz con relación a los modelos U-von Bertalanffy y U-Logístico, no hubo necesidad de estimar un modelo promedio.

TABLA 1

Parámetros de crecimiento, estadísticos y criterio de información de Akaike (AIC) de Plagioscion squamosissimus en el Orinoco medio, según las longitudes retro-calculadas en los anillos de crecimiento y las longitudes observadas

TABLE 1

Growth parameters, statistics and Akaike information criterion (AIC) of Plagoscion squamosissimus in the middle Orinoco, according to the retro-calculated lengths in the growth rings and the observed lengths

\begin{tabular}{|c|c|c|c|c|c|c|c|c|c|}
\hline & $L_{\infty}$ & $K$ & $T_{i}$ & $R^{2}$ & $W_{o}$ & $R S S$ & $A I C$ & $\Delta \mathrm{AIC}$ & $W_{i}$ \\
\hline \multicolumn{10}{|c|}{ Long. retro-calculadas } \\
\hline von Bertalanffy & $56.8(48.0-65.7)$ & $0.209(0.144-0.275)$ & 3 & 72 & 3 & 10995 & 644 & 231 & 0 \\
\hline Logístico & $58.1(57.2-58.9)$ & $0.683(0.648-0.718)$ & 4 & 81 & 7 & 7387 & 564 & 152 & 0 \\
\hline Gompertz & $60.0(54.8-65.2)$ & $0.104(0.090-0.110)$ & 3 & 95 & 6 & 1965 & 412 & 0 & 1 \\
\hline \multicolumn{10}{|l|}{ Long. observadas } \\
\hline von Bertalanffy & $59.3(54.9-63.7)$ & $0.190(0.150-0.231)$ & 4 & 91 & 2 & 3272 & 513 & 310 & 0 \\
\hline Logístico & $56.0(54.9-63.7)$ & $0.432(0.423-0.442)$ & 4 & 91 & 7 & 2950 & 472 & 269 & 0 \\
\hline Gompertz & $59.8(57.4-62.2)$ & $0.103(0.090-0.107)$ & 3 & 96 & 6 & 1485 & 203 & 0 & 1 \\
\hline
\end{tabular}

$L_{\infty}=$ Longitud asintótica $K=$ Máxima tasa de crecimiento relativo en la inflexión de la curva; $T_{i}=$ Edad en la inflexión de la curva; $W_{o}=$ Valor inicial de la curva $(t=0) ; R^{2}=$ Coeficiente de determinación; $R S S=$ Suma de cuadrado residual; AIC $=$ Criterio de información de Akaike; $W_{i}=$ Ponderación de Akaike.

El modelo U-Gompertz según los datos retro-calculados estuvo representado por la ecuación Longitud $=60.0 * \exp (-\exp$ $(-2.71828 * 0.104 *($ Edad-3.0) $))$; con una longitud inicial de la curva de $W_{o}=6.0 \mathrm{~cm}$ de longitud total y una edad para el punto de inflexión de $T_{i}=3.0$ años (Tabla 1; Fig. 1), mientras que según los datos observados el modelo U-Gompertz estuvo representado por la ecuación Longitud $=59.8 * \exp (-\exp (-$ $2.71828 * 0.099 *($ Edad-3.0) $))$, también con una longitud inicial de la curva de $W_{o}=6.0 \mathrm{~cm}$ de longitud total y una edad para el punto de inflexión de $T_{i}=3.0$ años; aparentemente sin diferencias significativas con relación a la curva de crecimiento estimada para los datos de longitud-edad retro-calculados (Fig. 1).

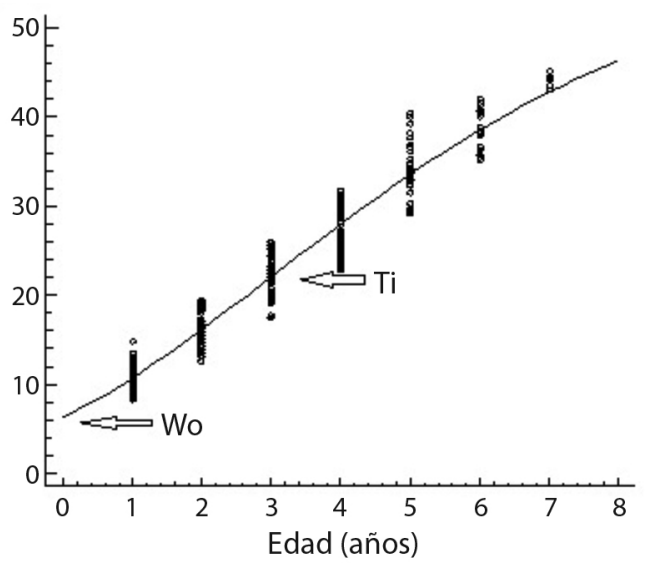

Fig. 1. Curva de crecimiento según el modelo U-Gompertz usando datos de longitud-edad retro-calculados.

Fig. 1. Growth curve according to the U-Gompertz model using retro-calculated length-age data. 


\section{DISCUSIÓN}

Una efectiva administración de $P$. squamosissimus en el Orinoco medio necesita de la evaluación previa de la población explotada, donde los modelos analíticos de evaluación requieren como información los parámetros de crecimiento y deben por lo tanto ser estimados de manera precisa para obtener resultados confiables (Khan \& Khan, 2017). En este sentido, una mejor opción es el uso de modelos múltiples de crecimiento para estimar dichos parámetros, en comparación con el uso a priori del modelo de von Bertalanffy (Katsanevakis \& Maravelias, 2008). En la selección del modelo de mejor ajuste también el uso del criterio de la información de Akaike (AIC) resulta ser más confiable que el coeficiente de determinación $\mathrm{R}^{2}$, por cuanto dicho coeficiente solo explica el grado de variación entre las variables mientras que el AIC utiliza la información KullbackLeibler (distancia $K-L$ ) como medida conceptual de la distancia relativa de un modelo dado con relación al que en realidad describe la información contenida en los datos (Burnham \& Anderson, 2002; Arzola-Sotelo, 2013).

Según el AIC el modelo U-Gompertz fue el que produjo el mejor ajuste de los datos longitud-edad de $P$. squamosissimus, posiblemente debido a que aproximadamente el $80 \%$ de dichos datos estuvo conformado por peces juveniles e inmaduros (Mesquita, Cruz, Hallwass, \& Isaac, 2019). Tal como se demostró para la especie Colossoma macropomum en algunas lagunas de la confluencia de los ríos Solimões y Japurá del estado de Amazonas en Brasil, donde los datos de longitud-edad fueron mejor ajustados por los modelos Logístico y de Gompertz, debido a la presencia en los datos de una mayor cantidad de peces juveniles (Costa et al., 2013). Aparentemente el modelo de von Bertalanffy produce un mejor ajuste en datos donde se encuentran representadas equilibradamente todas las edades, por cuanto facilita el modelaje del crecimiento desde las primeras edades hasta las que se aproximan a la de su tamaño máximo o asintótico; tal como se observó para $P$. orinocoense en el Orinoco medio (González et al., 2019).

Según estos resultados, aparentemente el modelo de mejor ajuste de datos de longitudedad no sólo depende del patrón de crecimiento de una determinada especie, sino que también influye la información que dichos datos contienen en cuanto a la distribución por edad; siendo el objetivo del AIC no solo modelar dichos datos, sino también poner de manifiesto esta información (Burnham y Anderson, 2002; Katsanevakis y Maravelias, 2008).

En este trabajo se demostró que en realidad el modelo que mejor se ajusta a los datos usados por González et al. (2005a) es el modelo U-Gompertz y no el de von Bertalanffy, creándose una incertidumbre en los valores de los parámetros de crecimiento que se estimaron $\mathrm{y}$, por lo tanto, en el resultado de una evaluación del stock de $P$. squamosissimus en la región del Orinoco medio que demostró la existencia de un recurso moderadamente explotado (González et al., 2005b). De allí que se recomienda evaluar nuevamente dicho stock para determinar realmente la condición en que se encuentra el recurso debido a la intensa explotación a la que ha estado sometido durante los últimos años, pero usando parámetros de crecimiento estimados desde una inferencia de modelos múltiples, aplicados a datos actualizados.

Declaración de ética: los autores declaran que todos están de acuerdo con esta publicación y que han hecho aportes que justifican su autoría; que no hay conflicto de interés de ningún tipo; y que han cumplido con todos los requisitos y procedimientos éticos y legales pertinentes. Todas las fuentes de financiamiento se detallan plena y claramente en la sección de agradecimientos. El respectivo documento legal firmado se encuentra en los archivos de la revista.

\section{AGRADECIMIENTOS}

Nuestros más sinceros agradecimientos a la Comisión de Investigación del Núcleo de Bolívar de la Universidad de Oriente, 
por el financiamiento del proyecto asociado a la publicación previa que originó el presente trabajo, así como a la Dirección del Instituto Limnológico de la Universidad de Oriente por la logística prestada.

\section{RESUMEN}

Introducción: El uso a priori del modelo de von Bertalanffy ha sido generalmente utilizado en el estudio del crecimiento en peces, sin considerar la existencia de otros modelos de crecimiento que pueden producir un mejor ajuste de los datos que se utilizan; considerándose como una mejor alternativa el uso de modelos múltiples de crecimiento, para seleccionar el que produzca un mejor ajuste. Objetivo: Ante la incertidumbre de los resultados obtenidos en un trabajo anterior sobre el crecimiento de Plagioscion squamosissimus (Heckel 1840) en la región del Orinoco medio en Venezuela, aplicando a priori el modelo de von Bertalanffy, los mismos datos fueron ajustados a otros modelos de crecimiento para entre ellos seleccionar el de mejor ajuste. Métodos: Los modelos evaluados fueron el U-von Bertalanffy, el U-Logístico y el U-Gompertz, derivados del modelo global U-Richards, entre los cuales se seleccionó el de mejor ajuste utilizando el Criterio de la Información de Akaike (AIC). Resultados: Contradiciendo el uso a priori del modelo de von Bertalanffy en el estudio anterior, el modelo que realmente produce un mejor ajuste de los datos usados fue el U-Gompertz. Conclusiones: Es necesario evaluar nuevamente el stock de P. squamosissimus en la región del Orinoco medio usando parámetros de crecimiento estimados a partir de una inferencia de modelos múltiples, para validar la información que se tiene en cuanto a la existencia de un recurso moderadamente explotado.

Palabras clave: peces de río; Orinoco medio; Plagioscion squamosissimus; dinámica de poblaciones; crecimiento; teoría de la información.

\section{REFERENCIAS}

Ansah, Y.B., \& Frimpong, A. (2015). Using model-based inference to select a predictive growth curve for farmed tilapia. North American Journal of Aquaculture, 77(3), 281-288.

Aragón-Noriega, E.A., Alcántara-Razo, E., ValenzuelaQuiñonez, W., \& Rodriguez-Quiroz, G. (2015). Multi-model inference for growth parameter estimation of the bigeye croaker Micropogonias megalops in the upper gulf of California. Revista de Biología Marina y Oceanografia, 50(1), 25-38.

Arzola-Sotelo, E.A. (2013). Aplicación de la teoría de modelos múltiples en la evaluación del crecimiento individual del chano (Micropogonias megalops, Gilbert 1890) en el norte del Golfo de California (Tesis de maestría). Centro de Investigaciones Biológicas del Noroeste, La Paz, México.

Burnham, K.P., \& Anderson, D.R. (2002). Model selection and multimodel inference: A practical informationtheoretic approach. New York, USA: Springer.

Costa, L.R., Barthem, R.B., Albernaz, A.L., Bittencourt, M.M., \& Villacorta-Corrêa, M.A. (2013). Modelling the growth of tambaqui, Colossoma macropomum (Cuvier, 1816) in floodplain lakes: model selection and multimodel inference. Brazil Journal Biology, 73(2), 397-403.

González, A., Mendoza, J., Arocha, F., \& Márquez, A. (2005a). Crecimiento de la curvinata de río Plagioscion squamosissimus, en el Orinoco medio. Zootecnia Tropical, 23(2), 155-170.

González, A., Mendoza, J., Arocha, F., \& Márquez, A. (2005b). Mortalidad y rendimiento por recluta de la curvinata de río Plagioscion squamosissimus en el Orinoco medio. Zootecnia Tropical, 23(3), 233-247.

González, A., Mendoza, J., Arocha, F., \& Márquez, A. (2016). Caracterización de la pesca artesanal en el río Orinoco sector Caicara-Cabruta, 2004-2008. Zootecnia Tropical, 34(1), 23-35.

González, A., Mendoza, J., Arocha, F., \& Márquez, A. (2019). Análisis multimodelo del crecimiento de Pseudoplatystoma orinocoense en la cuenca media del río Orinoco, Venezuela. Biotecnología en el Sector Agropecuario y Agroindustrial, 17(1), 1-24.

Katsanevakis, S., \& Maravelias, D. (2008). Modelling fish growth: multi-model inference as a better alternative to a priori using von Bertalanffy equation. Fish and Fisheries, 9(1), 178-187.

Khan, M.A. \& Khan, S. (2017). Precision of age estimates in striped snakehead Channa striata (Bloch, 1793) from the Ganga River and its tributaries (rivers Gomti and Yamuna). Journal Applied Ichthyology, 33(2), 230-235.

Mesquita, E.M.C., Cruz, R.E.A., Hallwass, G., \& Isaac, V.J. (2019). Fishery parameters and population dynamics of silver croaker on the Xingu River, Brazilian Amazon. Boletim do Instituto de Pesca, 45(2), e423. DOI: 10.20950/1678-2305.2019.45.2.423

Tjørve, E. \& Tjørve, K.M.C. (2010). A unified approach to the Richards-model family for use in growth analyses: Why we need only two model forms. Journal Theoretical Biology, 267(3), 417-425.

Tjørve, K.M.C., \& Tjørve, E. (2017). The use of Gompertz models in growth analyses, and new Gompertzmodel approach: An addition to the Unified-Richards. PLOS ONE 12(6): e0178691. DOI: 10.1371/journal. pone. 0178691 